Revue des patrimoines

46 | 2022

Le patrimoine de la Justice

\title{
Palais de justice et prisons départementales de Pau (1850-1865)
}

L'architecture des lieux de justice du département des Basses-Pyrénées à l'épreuve des enjeux locaux et nationaux

Courthouse and departmental prisons of Pau (1850-1865). The architecture of justice facilities in Basses-Pyrénées department to the test of local and national issues

\section{Cécile Devos}

\section{(2) OpenEdition}

Journals

Édition électronique

URL : https://journals.openedition.org/insitu/33412

DOI : 10.4000/insitu.33412

ISSN : 1630-7305

Éditeur

Ministère de la Culture

Référence électronique

Cécile Devos, «Palais de justice et prisons départementales de Pau (1850-1865) », In Situ [En ligne],

46 | 2022, mis en ligne le 24 janvier 2022, consulté le 03 février 2022. URL : http://

journals.openedition.org/insitu/33412 ; DOI : https://doi.org/10.4000/insitu.33412

Ce document a été généré automatiquement le 3 février 2022.

\section{(c) $(1) \odot$}

In Situ Revues des patrimoines est mis à disposition selon les termes de la licence Creative Commons Attribution - Pas d'Utilisation Commerciale - Pas de Modification 4.0 International. 


\title{
Palais de justice et prisons départementales de Pau (1850-1865)
}

L'architecture des lieux de justice du département des Basses-Pyrénées à l'épreuve des enjeux locaux et nationaux ${ }^{1}$

\author{
Courthouse and departmental prisons of Pau (1850-1865). The architecture of \\ justice facilities in Basses-Pyrénées department to the test of local and national \\ issues
}

Cécile Devos

1 Capitale de la vicomté souveraine de Béarn à partir de 1454, du royaume de Navarre de 1512 à 1620, la ville de Pau est dotée à cette date d'un parlement. En contrepartie de son entrée dans le royaume de France et du respect de ses principes de fonctionnement, la ville devient parlementaire; le Béarn conserve néanmoins ses fors, héritage judiciaire de sa souveraineté passée. La Révolution vient certes bousculer et repenser l'exercice de la justice pour affirmer l'indépendance de son pouvoir mais elle ne bouscule pas pour autant l'équilibre géographique de cet exercice en Béarn. L'ancien parlement devient palais de justice et Pau conserve sa prééminence dans le ressort de l'administration judiciaire nouvellement créée. Elle conserve également cette position, contestée au départ par d'autres villes, dans l'administration plus large de l'ancienne province, en devenant chef-lieu de département et siège de préfecture.

2 Capitale administrative ainsi confirmée, Pau connaît une nouvelle fortune dans les années 1830-1840 comme destination touristique prisée. Entre autres, le mythe de son beth $c e u^{2}$, son lien privilégié avec les stations thermales pyrénéennes, la publicité faite autour des vertus thérapeutiques de son climat par les militaires britanniques et en particulier le médecin Alexander Taylor ${ }^{3}$ ont pour conséquence une expansion géographique et démographique sans précédent. Désormais terre d'accueil pour populations phtisiques de tous horizons ou hivernants en quête de plaisirs et de bonne société, la ville, le département et l'État accompagnent la croissance du territoire et encadrent strictement l'exercice de son administration. Cet élan passe par l'engagement d'une réflexion commune aux différentes administrations sur les 
infrastructures nécessaires au développement d'une telle ville, sur l'architecture publique et son intégration dans le tissu urbain. Cette réflexion locale s'inscrit dans le cadre de préoccupations nationales.

C'est dans ce mouvement général de transformation du paysage palois, vers le milieu du XIX ${ }^{e}$ siècle, que les autorités judiciaires et pénitentiaires s'attellent à la construction d'un nouveau palais de justice puis de nouvelles prisons départementales. L'implantation et l'architecture de ces établissements font l'objet d'une patiente élaboration, mise en œuvre par les deux architectes départementaux successifs de la période. Entre recommandations nationales et courants de pensée architecturaux, ces deux lieux, qui ont conservé leur usage premier jusqu'à nos jours, expriment à leur manière une adaptation pragmatique aux ressources disponibles dans leur mise en œuvre comme les lointains débats dont ils se font l'écho. Ils permettent aussi de situer Pau sur la carte des administrations «bonnes élèves » dans le relais du pouvoir central, signe d'un intérêt réciproque à favoriser et rendre possible la mise en application de « l'intérêt général » en aménagement urbain.

\section{Une ville en ébullition}

\section{Projets monumentaux en litanie}

4 "Que de choses à faire : Messieurs! Faut-il se laisser décourager. Non, mille fois non, il faut au contraire retremper son courage à la nécessité de sauver la ville, en procédant toujours, mais sans folle précipitation à des améliorations utiles ${ }^{4}$. " Voilà un résumé de l'état d'esprit dans lequel les édiles palois travaillent à la construction de la ville tout au long $\mathrm{du} \mathrm{XIX}^{\mathrm{e}}$ siècle. Durant sa première moitié, Pau voit naître de nouvelles administrations qui s'installent, faute de mieux, entre les murs des anciennes ${ }^{5}$. Ces enceintes sont souvent vétustes et ne sont pas en mesure de répondre aux nombreux besoins. Lieux de justice, sièges des administrations locales, places et marchés, églises paroissiales, l'espace public et les monuments palois font l'objet de nombreuses discussions, parfois suivies de mise en œuvre. Le palais de justice est établi assez naturellement dans l'ancien parlement, transformation éminemment honorable dans le sens, mais peu adaptée dans les capacités d'accueil et de travail. L'ancien hôtel du Premier président au parlement devient l'hôtel de préfecture. Comme partout, les biens nationaux sont massivement réinvestis pour accueillir de nouvelles fonctions. Par exemple, l'ancien couvent des Cordeliers abrite pour un temps l'hôtel de ville, l'ancien couvent des Filles de la Foi devient temporairement une caserne, etc. Les prisons quittent le château pour être hébergées dans l'ancien hôtel de la famille parlementaire des Gassion, racheté et restauré par le département à cet effet et situé contre l'ancien parlement, devenu palais de justice.

L'entrée nord-ouest de la ville, par la place Gramont, voit enfin l'aboutissement de ses travaux, entamés dans les années 1780, avec l'achèvement des talus de la place, en 1840-1841. La caserne Bernadotte sort de terre et peut recevoir ses premiers soldats dès 1831 mais ses travaux sont loin d'être terminés. Nouveau centre, au sein d'un îlot auparavant composé de l'agglomération de différents couvents, une nouvelle halle abritant étaliers et services municipaux est envisagée dès 1820 puis construite et ouverte en 1852. Le projet d'achèvement des travaux de Saint-Louis, église bordant la place Royale commencée à la fin du $\mathrm{XVII}^{\mathrm{e}}$ siècle, est à nouveau évoqué sans que ne 
pointe l'ombre d'une réelle reprise de chantier. Les deux églises paroissiales, SaintJacques (ancienne chapelle des Cordeliers) et Saint-Martin (première église paroissiale), accusent le poids des ans. Leur remplacement, dans le fonds et la forme, est envisagé dans cette première moitié du siècle pour être mis en œuvre après 1850 , afin de pourvoir aux besoins religieux de la population, en plein accroissement ${ }^{6}$ [fig. 1].

Figure 1

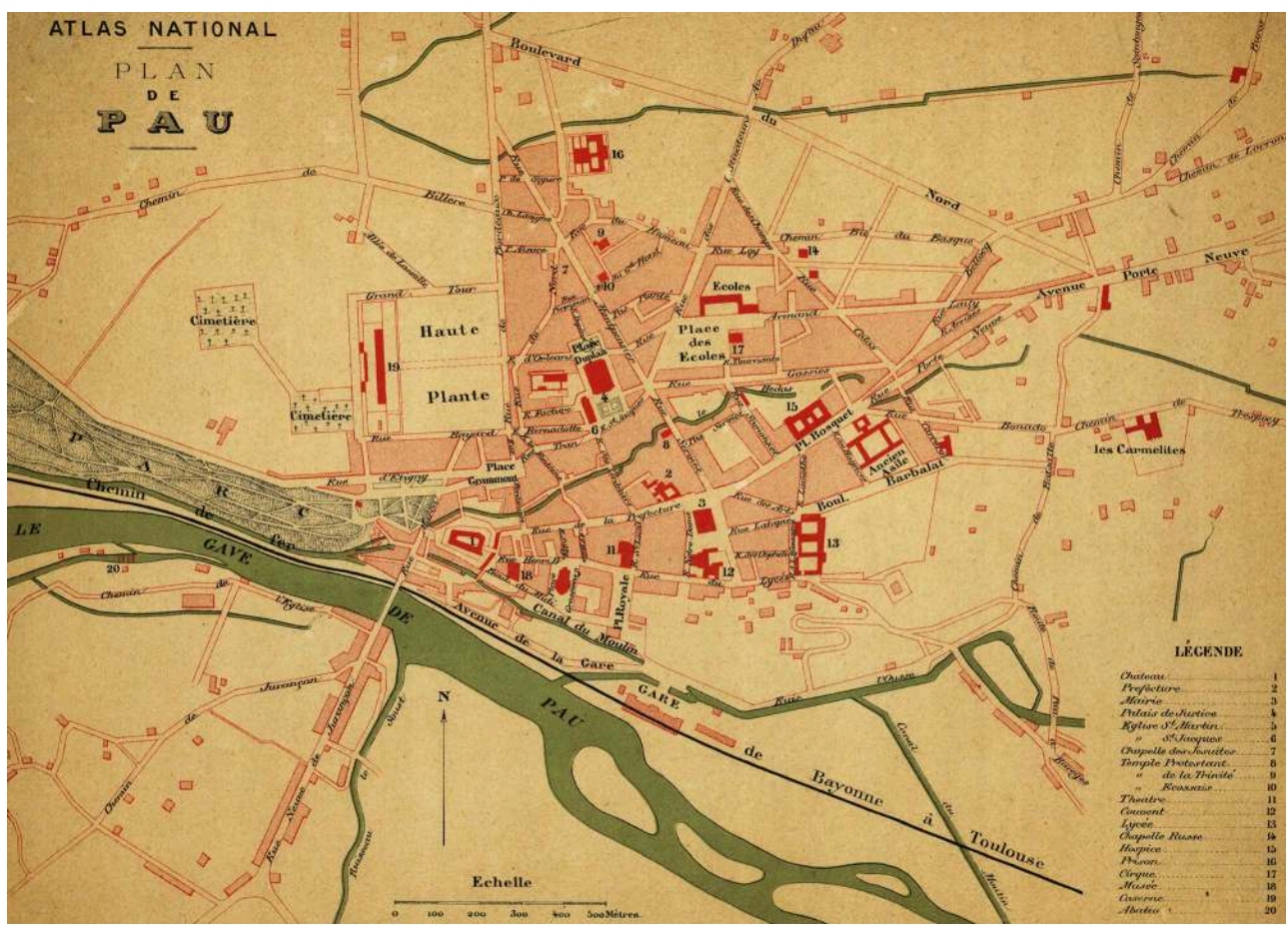

Atlas national. Plan de Pau, Hausermann Rémy graveur, s. d., conservé aux archives communautaires de Pau-Béarn-Pyrénées (5Fi1).

Reproduction archives communautaires de Pau-Béarn-Pyrénées.

\section{En filigrane, une réflexion sur la ville}

Les biens nationaux ont offert des opportunités architecturales rapidement disponibles mais souvent inadaptées aux nouveaux usages. Ils fournissent surtout des emprises parcellaires qui favorisent la naissance d'une réflexion sur les transformations nécessaires et possibles pour accompagner la croissance du tissu urbain. Les projets témoignent d'un air du temps où architecture et aménagement urbain sont désormais examinés de pair, au niveau local comme national par le biais du Conseil des bâtiments civils. La démolition du séminaire favorise la création, au tournant du siècle, de la rue Serviez, dégagement du centre-ville vers le nord devenu indispensable. L'état des lieux établi lors de la venue de Napoléon en 1808, qui tente de relancer quelques projets urbains locaux en suspens ${ }^{7}$, rend compte des débats en cours : nécessité d'un hôtel de ville, extension de la place Royale et ouverture vers le paysage... La mise en œuvre de ces déclarations d'intention prend un certain temps à Pau mais aboutit vers les années 1840 , avec la réalisation de plans de ville ${ }^{8}$ et l'érection des nouveaux bâtiments publics aux abords systématiquement redessinés. 
7 Délibérations, articles de presse ou décrets rendent compte des discussions qui ont pour objet ce qu'ils nomment «l'embellissement urbain», au travers des projets de construction publique. Chaque construction nouvelle doit en porter une part, et l'architecture publique plus encore. Au départ d'un projet architectural, la réflexion sur ses formes entraîne une réflexion sur son insertion dans le paysage, sur ses rapports au centre, mouvant à Pau, sur les liens qu'il entretient avec ses semblables, l'important restant de compter de beaux monuments et les plus nombreux possible. À propos du palais de justice, il est dit par exemple que «ce sera une construction vraiment monumentale, qui contribuera puissamment à l'embellissement de la ville de $\mathrm{Pau}^{9}$ ", ce qui témoigne du double enjeu architectural et urbain. Est également interrogée la notion même de centre-ville. Où se situe-t-il vraiment? Autour du château ? De la place Royale? Les concepteurs de la place Gramont ont voulu en faire un nouveau pôle de la cité, ambition qui ne s'est pas concrétisée. La nouvelle halle pourrait aussi en devenir un. En ces temps de multiplication des projets, la construction de chaque nouveau monument relance le débat.

8 Ces réflexions s'intensifient au milieu du siècle lorsque le département des BassesPyrénées interroge le conseil municipal sur la possibilité de construire un nouveau palais de justice dans la ville, élément déclencheur du déménagement des prisons et de transformations urbaines.

\section{Palais de justice et prison, du projet à la réalisation}

\section{Le palais de justice, site et architecture ${ }^{10}$}

9 Le conseil municipal palois écarte vite la possibilité de subvenir financièrement à la demande en achetant un terrain, un hôtel parlementaire étant proposé à la vente. Il préfère examiner les possibilités foncières offertes par les propriétés publiques, l'ancien couvent des Cordeliers servant d'hôtel de ville ou l'îlot des Ursulines et Orphelines. La superficie généreuse de l'ancien couvent, $10000 \mathrm{~m}^{2}$, est un argument de poids. Le projet initial de la commission chargée d'examiner les possibilités d'édification du nouveau bâtiment évolue vers une prise en considération d'un nécessaire aménagement urbain. Ainsi, le don d'un terrain conséquent au maître d'ouvrage est assorti de l'obligation de créer de nouvelles voies favorisant des dégagements futurs. La rue d'Orléans est percée en 1845, alors que le projet de palais de justice est en gestation, afin de permettre un contournement du centre-ville desservant les routes de Tarbes, de Bordeaux et de Bayonne. L'aménagement des abords du palais est approuvé en Conseil des bâtiments civils en 1848, car il respecte les « convenances d'isolement et de circulation ${ }^{11}$ ». Par ailleurs, le sacrifice de l'emprise des Cordeliers est accompagné dans un deuxième temps par l'achat de maisons ou terrains de particuliers, ce qui n'avait pas été initialement prévu ${ }^{12}$. Des places ont été envisagées dès le départ, au nord comme au sud de l'édifice, écrins que le conseil municipal tend à présenter comme indispensables au monument ${ }^{13}$. La place nord et la rue qui la prolonge dans ce sens nécessitent plus d'acquisitions pour leur établissement que prévu. Le projet, mené à son terme, évolue pour mettre en scène le nouvel édifice en profitant du dénivelé et en en dégageant les entrées par la création de rues plus larges, les actuelles rue Mourot et Faget de Baure [fig. 2]. 
Figure 2

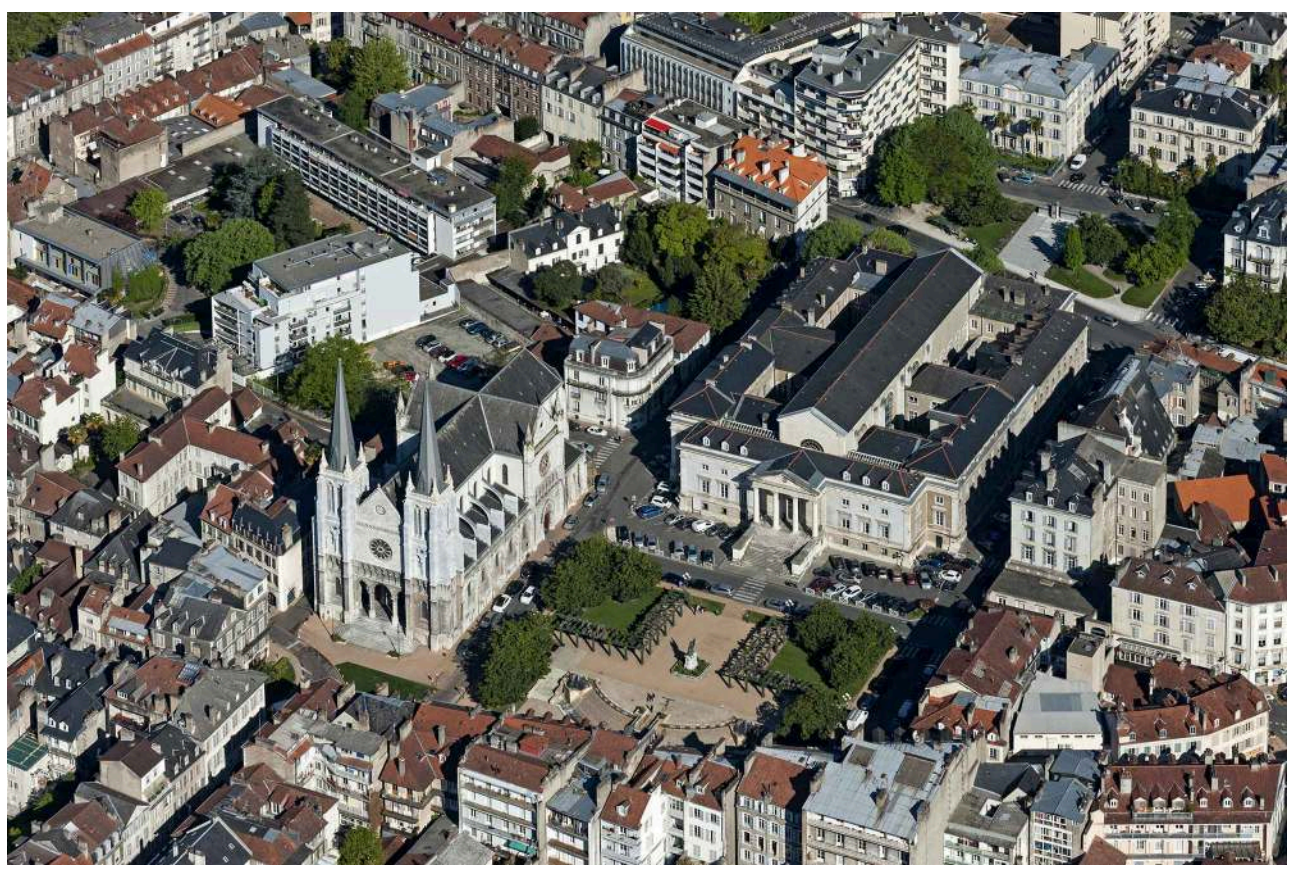

Le palais de justice de Pau dans son quartier, 2013.

(c) Marc Heller (Communauté d'agglomération de Pau-Béarn-Pyrénées).

10 La commission municipale souligne à raison qu'elle n'a pas d'avis à donner concernant la forme du palais de justice à construire, effectivement sous la responsabilité du département et le contrôle du Conseil des bâtiments civils. C'est ainsi que Vincent Latapi $^{14}(1797-1860)$, architecte du département, s'empare du projet et en dessine les contours. Un premier envoi de plans au Conseil est daté de mars 1846. Les principaux éléments du projet sont dressés dès cette époque : plan allongé avec distribution en peigne des salles d'audience, rez-de-chaussée « technique », entrée au niveau supérieur par un péristyle à quatre colonnes, vestibule puis salle des pas perdus voûtée en berceau dans l'axe de l'entrée pour l'accès aux salles d'audience, deuxième étage consacré principalement aux bureaux. En avril 1847, un second envoi apporte quelques ajustements, notamment en rez-de-chaussée, avec la réduction du nombre de cellules et la modification du système de fondations, puis du voûtement de la salle des pas perdus et de la cour d'assises. L'évidement des coins du rectangle d'ensemble mis en œuvre n'apparait pas encore dans ces dessins, preuve qu'il y eut d'autres révisions apportées par la suite. La pose de la première pierre a lieu le 8 novembre $1847^{15}$, le palais de justice étant inauguré le 3 janvier 1856. L'ajout d'un vitrail et la modification d'une des grandes salles datent des années 1860. Deux statues, consacrées à des figures tutélaires de la justice béarnaise, Pierre de Marca (1594-1662) et Jean-François Régis Mourot (1740-1813), auraient pu venir compléter et encadrer l'entrée monumentale. Ce projet de 1878 n'est pas réalisé faute de moyens ${ }^{16}$.

Les architectes reprennent les principaux éléments du type de palais de justice tel que conçu et identifiable en France depuis la fin du XviII ${ }^{e}$ siècle $^{17}$. L'architecture du palais de justice de Pau respecte de grands principes généraux que le Conseil des bâtiments civils préconise, diffuse et encadre pour toute architecture publique : emploi des ordres à des parties bien déterminées de l'édifice [fig. 3 et fig. 4], hiérarchisation du décor en fonction de l'importance de l'édifice, adaptation aux réalités du programme et à 
l'usage ${ }^{18}$. Pour les palais de justice plus spécifiquement, les avis du Conseil tendent à privilégier le "caractère ", c'est-à-dire privilégier l'emploi traditionnel des ordres, l'articulation de volumes géométriques simples, un décor rare, une " éloquence simple, presque romaine ", qui soulignent à l'économie le caractère de l'édifice et mettent en scène la justice ${ }^{19}$.

Figure 3

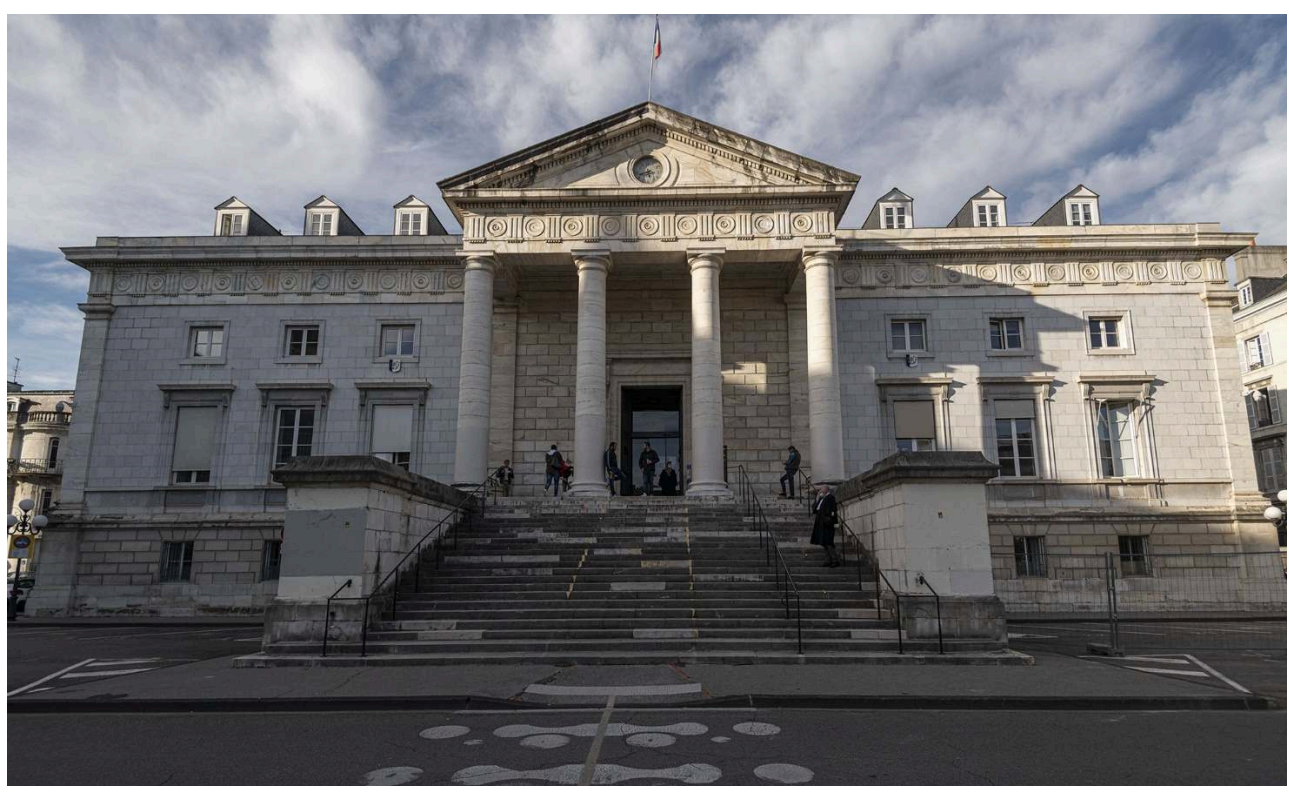

Façade méridionale du palais de justice de Pau, 2020

(c) Sébastien Arnouts (Ville de Pau, Région Nouvelle-Aquitaine, Inventaire général)

Figure 4

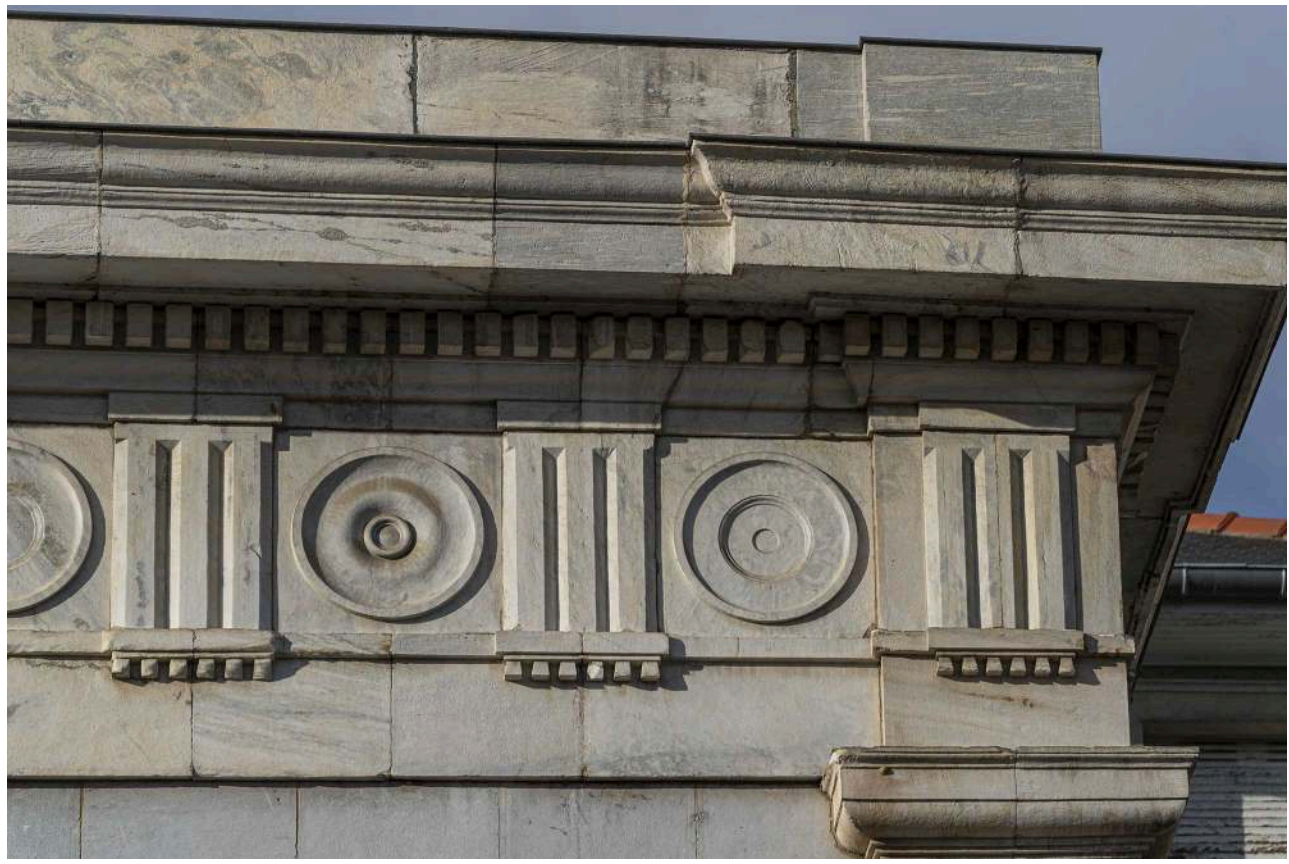

Détail d'entablement de la façade sud du palais de justice de Pau, 2020.

(C) Sébastien Arnouts (Ville de Pau, Région Nouvelle-Aquitaine - Inventaire général). 
12 Le palais palois respecte la totalité de ces orientations [fig. 5 et fig.6]. Comme de nombreux autres, il a pu prendre modèle sur le palais de justice d'Angoulême (Charente, 1825-1828) de l'architecte Paul Abadie père (1783-1868), publié et diffusé comme source d'inspiration sous la houlette du Conseil des bâtiments civils dans le recueil de Charles Gourlier ${ }^{20}$.

\section{Figure 5}

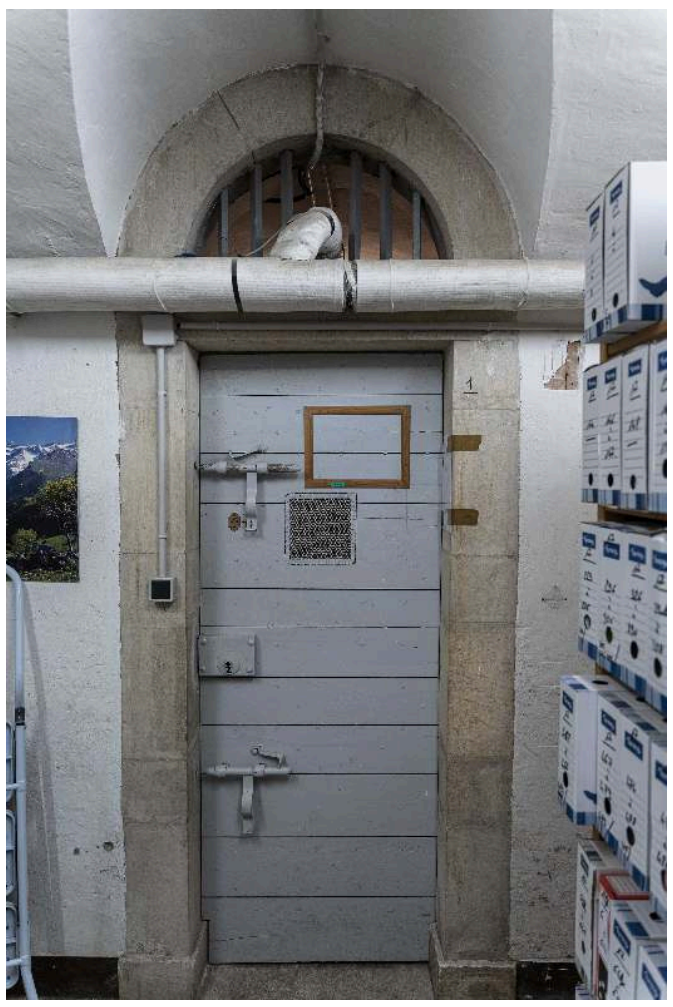

Ancienne geôle, rez-de-chaussée du palais de justice de Pau, 2020.

(c) Sébastien Arnouts (Ville de Pau, Région Nouvelle-Aquitaine, Inventaire général) 
Figure 6

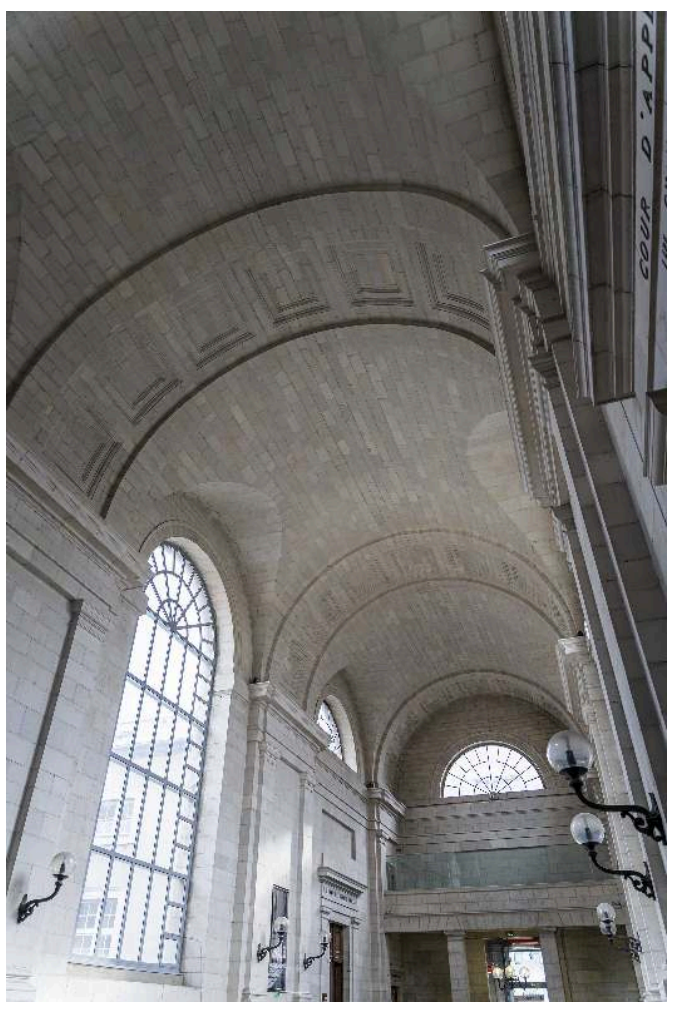

Voûtement de la salle des pas perdus du palais de justice de Pau, 2020.

(c) Sébastien Arnouts (Ville de Pau, Région Nouvelle-Aquitaine, Inventaire général).

\section{Son « accessoire nécessaire ", les prisons départementales ${ }^{21}$}

«L'accessoire nécessaire d'un palais de justice, ce sont des prisons ${ }^{22}$.» Lors de la construction du palais de justice, les prisons départementales sont hébergées depuis la fin du Premier Empire dans un ancien hôtel particulier du XVII ${ }^{\mathrm{e}}$ siècle réaménagé pour l'occasion, dans le centre ancien de la ville. Les constats montrent que les transformations successives de l'édifice ne sont pas totalement efficientes :

Tous les inspecteurs sont unanimes dans leurs plaintes. Les prisons ont plusieurs étages, disposition contraire aux premières règles de la salubrité : elles sont insuffisantes pour recevoir les condamnés des autres arrondissements, qui devraient d'après la loi, être réunis au chef-lieu. Les prisonniers n'ont pas de préaux; car on ne saurait donner ce nom à d'étroits corridors, aussi malsains que l'intérieur même de l'édifice. Quant à la séparation des prisonniers, telle qu'elle devrait exister, malgré tous les efforts de l'administration, elle est loin d'être complète. Or tout l'espace disponible est employé. Insuffisant avec le système actuel, que sera-t-il avec le régime cellulaire; et ce régime ne saurait manquer de prévaloir; le principe en étant généralement adopté, et la discussion ne portant plus que sur l'isolement absolu ou partiel ${ }^{23}$.

Les édiles profitent de la discussion engagée avec le département pour faire passer d'autres messages : celui du déménagement des prisons leur importe en priorité et leur avis semble éclairé par ces rapports réguliers et une certaine connaissance des débats contemporains sur la séparation des détenus et l'architecture carcérale, qui tend dans cette période à ériger l'emprisonnement cellulaire comme norme ${ }^{24}$. Au départ, ils pensent même pouvoir réserver un espace pour les prisons et une caserne de 
gendarmerie dans le terrain finalement octroyé au seul palais de justice: le lien fonctionnel existant entre eux est bien réel et l'association des deux largement mise en œuvre sur le territoire national ${ }^{25}$. La distance entre eux doit être la plus courte possible, pour que le chemin du prisonnier entre le jugement et la sentence en soit au maximum raccourci, pour prévenir également les troubles populaires qui pourraient venir émailler ce parcours. Ils "offrent" donc d'autant plus facilement le terrain des Cordeliers au départ du projet. En 1856, et alors que la construction du palais de justice s'achève, le conseil municipal décide d'interpeller plus fermement le préfet et le conseil général sur le sujet, «pour le vote de la construction immédiate des prisons ${ }^{26}$ ». Leurs motivations sont autant humanistes - améliorer le sort des détenus - qu'intéressées, le terrain sur lequel sont situées les prisons ayant pris de la valeur avec l'aménagement programmé d'une promenade à leurs pieds, le boulevard du Midi, équipement obligé pour toute station climatique honorable. La délicate équation, « mettre à distance tout en maintenant sous main de justice ${ }^{27}$ ", est en voie de résolution.

Léménagement est effectivement programmé et les prisons départementales sont édifiées à distance raisonnable du palais de justice, à environ 500 mètres au nord ${ }^{28}$, mais finalement reléguées hors du centre-ville, à dessein [fig. 7]. Construites entre 1863 et 1866, elles sont alors implantées dans un quartier dont le développement débute à peine et sont tout à fait isolées. Le chemin Lavigne, actuelle rue Viard, est ouvert à cette occasion pour desservir leur entrée unique, à l'ouest.

Figure 7

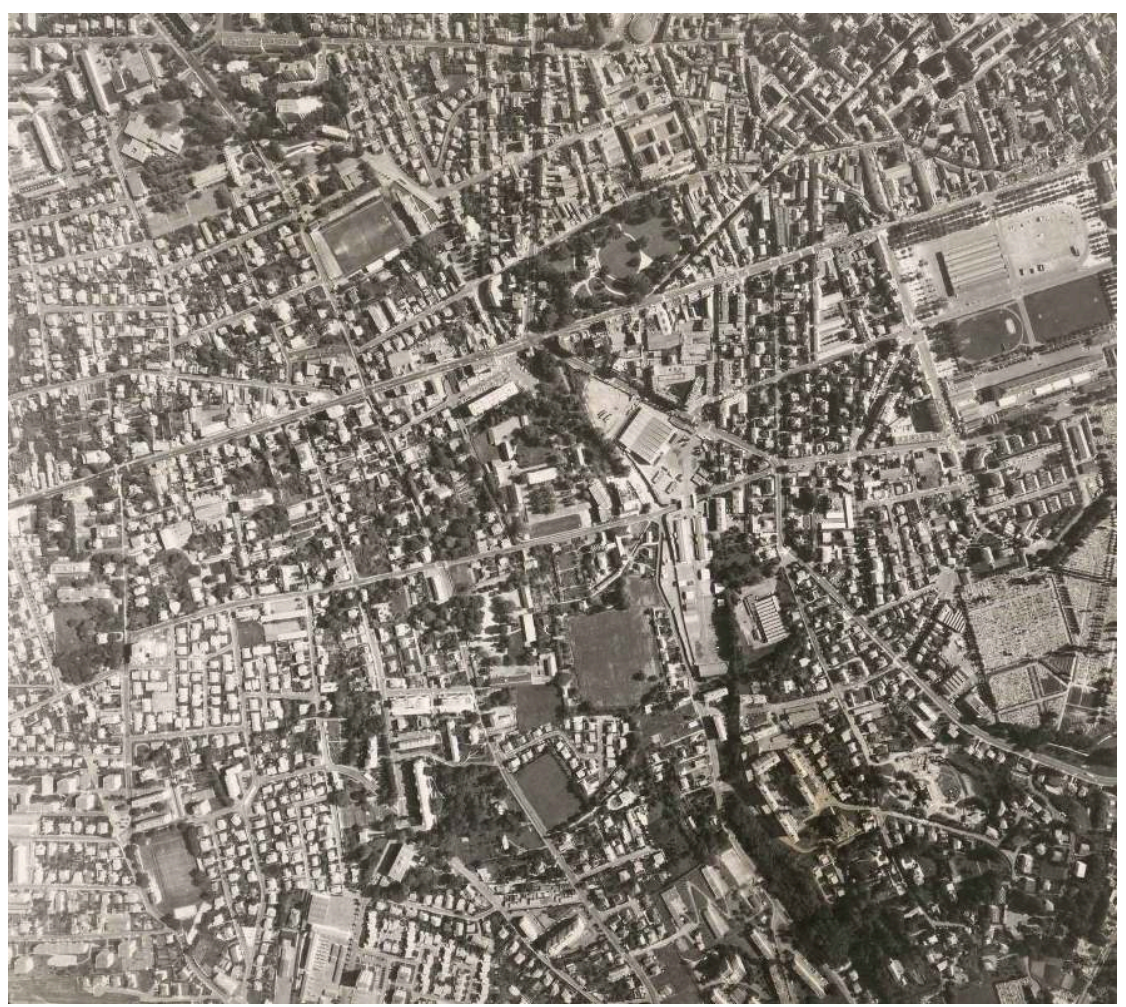

Quartier de la prison de Pau, en haut au centre de la vue aérienne, 1975, conservée aux archives communautaires de Pau-Béarn-Pyrénées (19Fi776).

(c) Photographe inconnu (ministère de l'Équipement, division des travaux topographiques). 
Le programme palois enclot dans une enceinte et un chemin de ronde un bâtiment qui, à l'instar du palais de justice, évide un carré en différents corps bâtis disposés en grille, alternant avec des préaux. Près de l'entrée unique se trouvent les services administratifs accueillant personnel et détenus, qui sont prolongés vers l'ouest avec le développement des secteurs réservés aux détenus séparés par sexe, âge et type de peine, et qui sont construits de manière similaire: rez-de-chaussée comprenant chauffoirs, réfectoires et ateliers, quelques chambres individuelles, puis à l'étage, chambres communes et individuelles. La chapelle, aujourd'hui disparue, se trouvait dans la partie centrale de l'édifice. Le programme de la prison paloise semble respecter celui de la circulaire du 17 août 1853, dite circulaire Persigny. Elle permet notamment la séparation des détenus par catégories, l'emprisonnement cellulaire, gourmand en fluides et en espace, n'étant plus autant d'actualité. Les Projets spécimens pour servir à la construction des prisons départementales, publiés en 1854, en sont un instrument de diffusion $^{29}$ [fig. 8].

Figure 8

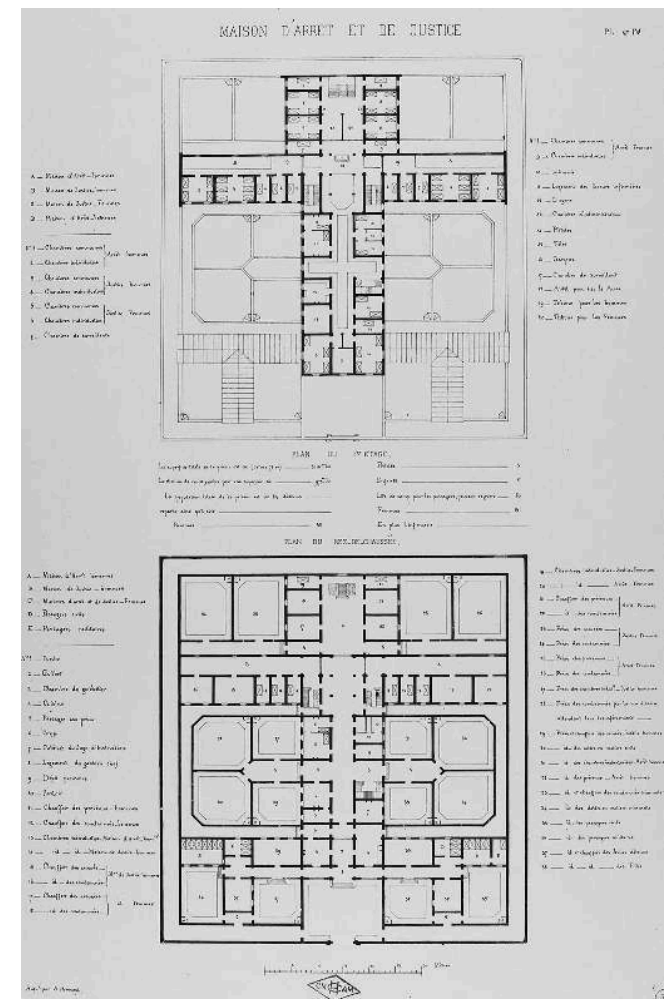




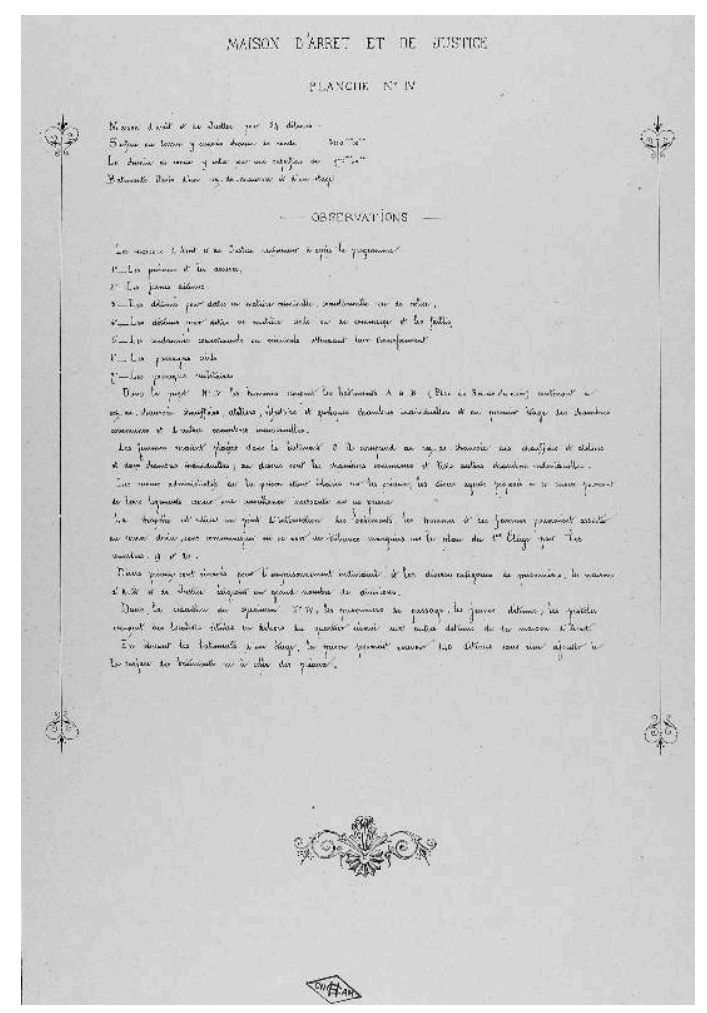

Maison d'arrêt et de justice pour 84 détenus, modèle théorique extrait de Projets spécimens pour servir à la construction des prisons départementales, 1854, conservé à la bibliothèque centrale du Conservatoire national des arts et métiers à Paris (Poupée A1).

Reproduction bibliothèque centrale du Conservatoire national des arts et métiers, Paris.

Ce recueil a été conçu par les architectes et membres du Conseil des bâtiments civils Edme Jean Louis Grillon (1786-1854) et Alfred Normand (1822-1909); ce dernier est l'auteur des dessins et est nommé en 1861 inspecteur des bâtiments pénitentiaires. Un des modèles pouvant avoir inspiré les prisons paloises figure dans le recueil, en planche 4. Si l'échelle est différente (le modèle est prévu pour $5000 \mathrm{~m}^{2}$ quand la prison paloise mesure presque $9000 \mathrm{~m}^{2}$ ), le programme semble équivalent. Il s'éloigne des expériences d'architecture carcérale et des plans rayonnants de la première moitié du $\mathrm{XIX}^{\mathrm{e}}$ siècle, pour répondre pragmatiquement à la construction d'une prison moyenne sur un plan presque carré, avec vie en communauté, travail, prière et surveillance mises en œuvre selon une attention égale.

18 La construction contemporaine du palais de justice et des prisons à Pau et leur très relatif éloignement viennent illustrer, s'il en était besoin, l'analyse plus récente de Michel Foucault :

Désormais, le scandale et la lumière vont se partager autrement; c'est la condamnation elle-même qui est censée marquer le délinquant du signé négatif et univoque : publicité donc des débats, et de la sentence ; quant à l'exécution, elle est comme une honte supplémentaire que la justice a honte d'imposer aux condamnés ; elle s'en tient donc à distance, tendant toujours à la confier à d'autres, et sous le sceau du secret. Il est laid d'être punissable, mais peu glorieux de punir. De là ce double système de protection que la justice a établi entre elle et le châtiment qu'elle impose. L'exécution de la peine tend à devenir un secteur autonome, dont un mécanisme administratif décharge la justice; celle-ci s'affranchit de ce sourd malaise par un enfouissement bureaucratique de la peine ${ }^{30}$. 


\section{Pau-Paris, 1845-1865}

19 Les édiles palois portent dans ces années-là une réflexion et une action qui contribueront à bouleverser le paysage de Pau. Ils évaluent les besoins en monuments publics et raisonnent en matière d'espaces et de ressources disponibles pour accueillir des édifices utiles, beaux et propres à assurer le service public et à donner la meilleure image possible de la ville, tourisme oblige. Ils construisent la ville contemporaine. Leur action doit tendre à encourager l'implantation de bâtiments neufs, dont ils ne tireront que des bénéfices en termes d'usage et d'esthétique. En même temps qu'ils favorisent l'implantation du palais de justice, ils appellent de leurs vœux le déplacement des prisons, décision qui ne leur appartient pas mais dont l'éloignement leur conviendrait. Ils réfléchissent ensuite à leurs propres priorités édilitaires suivantes : un hôtel de ville, puis, plus tard, une et deux églises. Il convient de noter que durant cette période, ils réussissent à éloigner du centre les potentielles nuisances liées aux prisons ou à l'asile d'aliénés ${ }^{31}$, tous deux sous la responsabilité du département et dont les bâtiments sont contemporains. Les sources locales montrent cette ferme volonté des responsables municipaux; ils semblent porter une vision d'ensemble dont l'objectif final est de pourvoir aux besoins en qualité d'hébergement des institutions, avec une certaine idée de la ville. Pourtant, les sources nationales, elles, tendent à montrer que les projets sont examinés par le Conseil au coup par coup, monument par monument ${ }^{32}$. L'envoi des dossiers palois au Conseil est échelonné : palais de justice, prisons, asile d'aliénés, églises, abords... quand d'autres villes ont réussi à regrouper leurs demandes : entre autres exemples, les Eaux-Bonnes (Pyrénées-Atlantiques) font conjointement examiner, le 26 avril 1864, leur projet associant établissement thermal, hôtel de ville, église et promenoir, équipements mis au service de la station thermale ${ }^{33}$.

L'unification de l'architecture publique passe plutôt par les instruments de diffusion des idées architecturales à disposition des maîtres d'œuvre, auxquels les maîtres d'ouvrage sont visiblement sensibilisés et réceptifs durant les années où ces instruments ont été éprouvés. Les édifices du palais de justice, de la prison ou de l'asile d'aliénés, réalisés par les architectes départementaux, suivent les préconisations nationales orchestrées et relayées notamment par le Conseil des bâtiments civils. Dans ce sens, Vincent Latapie et Gustave Lévy (1826-1885), architectes départementaux (qui parfois travaillent également pour la Ville), montrent une capacité d'assimilation et d'interprétation des modèles nationaux alors étudiés, recommandés et en cours de diffusion. Le Conseil des bâtiments civils n'intervient pas tant en gendarme qu'en modérateur et... conseiller des projets palois. Gustave Lévy notamment, connu pour ses nombreuses interventions locales et départementales, est particulièrement sollicité. Architecte des prisons et de l'asile d'aliénés, à l'instar de son voisin et contemporain Jean-Jacques Esquié ${ }^{34}$ (1817-1884) en Haute-Garonne ${ }^{35}$, il applique à ces deux créations les mêmes grands principes généraux relatifs à l'architecture publique de l'époque, l'aérisme de leurs dispositions permettant dans l'un comme dans l'autre de prévenir les troubles et contaminations liés aux maladies et à la promiscuité. Dans l'air du temps également, le travail, "moteur et repère des transformations individuelles ${ }^{36}$ ", est considéré comme utile aux prisonniers comme aux aliénés; asiles et prisons doivent donc comprendre de nouveaux espaces pour pouvoir l'accueillir. À Pau, pour des raisons touristiques locales mais suivant une tendance nationale, les édiles préfèrent déloger du centre, éloigner et construire de neuf ces «architecture[s] de la périphérie ${ }^{37}$ " dans des quartiers vierges et en devenir, sans pour instant les exclure 
totalement de la ville de laquelle ils participent pleinement. Soulignons que Gustave Lévy est un élève de Guillaume-Abel Blouet à l'école des Beaux-Arts et certainement très au fait de l'actualité architecturale et théorique, ne serait-ce qu'en suivant les publications de son ancien professeur sur l'architecture carcérale.

Par ailleurs, il faut retenir l'empressement des «Basses-Pyrénées » de l'époque à se doter des équipements dont la mise au goût du jour ou la construction sont fortement conseillées et encouragées par l'État et à suivre les directives nationales; ce n'est pas forcément le cas dans tous les départements mais on peut observer ici une belle continuité entre la Monarchie de Juillet et le Second Empire. Ce partenariat politique et administratif trouve-t-il son explication dans les qualités des personnages aux ambitions locales et nationales qui se succèdent à la tête du département dans ces années de ferveur édilitaire, les préfets Jules Azévédo (1795-1860) et Armand Laity (1812-1889) et les présidents du conseil général des Basses-Pyrénées Pierre de SaintCricq (1772-1854) et Auguste Dariste (1807-1875) ? Dans l'attention stratégique portée à la paix civile et à ses instruments dans le département, à la fois côtier et frontalier ? Sans réponse à ce stade de l'étude, il convient de noter que l'écho rencontré auprès des édiles palois, soucieux de l'attractivité de leur ville et de son développement économique, y est alors évidemment favorable.

\section{Et demain?}

Figure 9

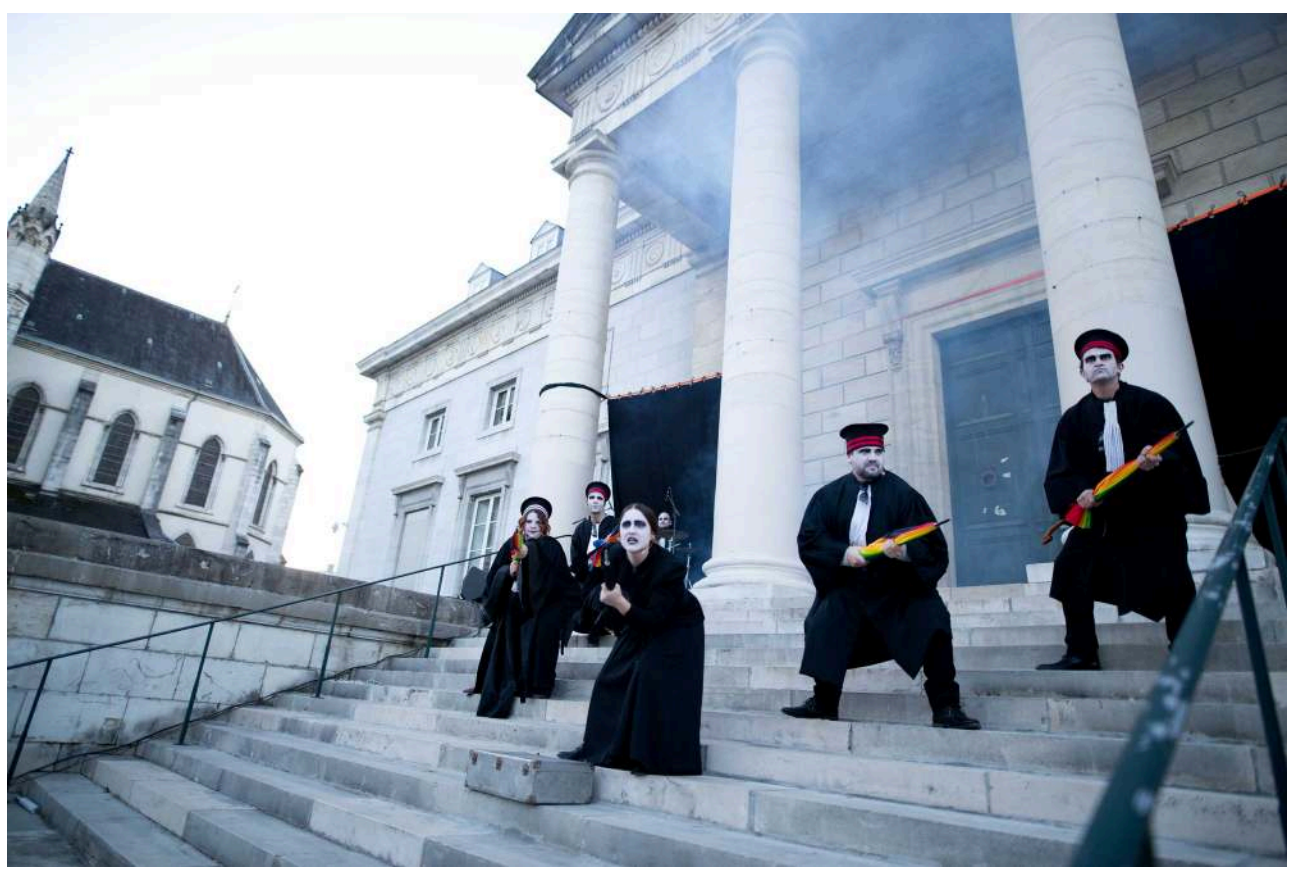

Parodie de justice durant le Carnaval béarnais, Pau, 2018

(c) Cyril Garrabos (Ville de Pau).

L'administration en charge des deux établissements étudiés ici a très sérieusement pris en main leur ouverture aux publics et leur valorisation. [fig. 9] Prison et palais de justice sont tous deux intégrés dans le site patrimonial remarquable de la ville; ils ne bénéficient pourtant pas du même degré de protection ni du même intérêt. Intérieurs 
et extérieurs du palais de justice sont protégés par la servitude, alors que ceux de la prison, dans un quartier signalé comme secteur de projets, en sont oubliés. Les usages actuels apparaissent souvent comme difficilement compatibles avec la conservation patrimoniale, quand bien même elle s'attache à ses éléments les plus qualitatifs (menuiseries, décor sculpté ou peint voire mobilier...) ; la tendance générale est à la réforme plus qu'à la restauration. La conservation d'un vitrail du Christ en croix (1866) $\mathrm{du}$ maître verrier parisien Claudius Lavergne (1815-1887), dans une salle d'audience, fait depuis quelques années l'objet de débats et d'aménagements [fig. 10]. Sa présence dans son écrin d'origine ne peut plus être remise en cause depuis l'intégration du bâtiment dans le Plan de sauvegarde et de mise en valeur mais sa visibilité pourrait, elle, faire l'objet de dispositifs destinés à cacher temporairement la représentation religieuse aux yeux du public le temps des audiences, comme aujourd'hui, un rideau cachant la verrière. Si cette solution a l'avantage d'éviter la dispute, elle a aussi l'inconvénient de renvoyer à plus tard l'explication de texte sur la présence d'un tel objet dans l'administration judiciaire, à la valeur patrimoniale bien réelle.

Figure 10

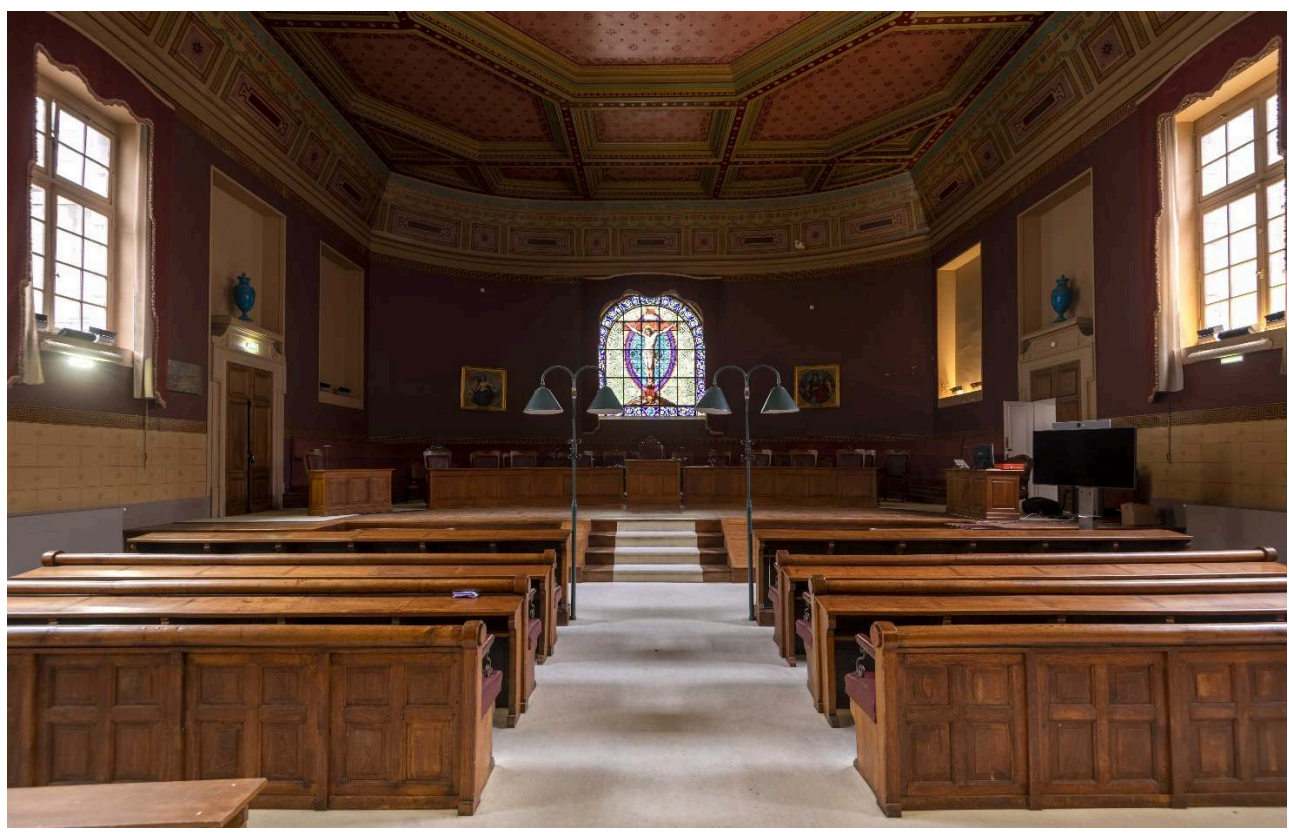

Vitrail de l'ancienne chambre civile du palais de justice représentant le Christ en croix, Pau, 2020.

(c) Sébastien Arnouts (Ville de Pau, Région Nouvelle-Aquitaine, Inventaire général).

Dans le cadre d'une éducation citoyenne, il est tout à fait possible d'assister à une audience dans le palais de justice ou d'intégrer la visite et la compréhension du site et de son fonctionnement dans un projet pédagogique, sous réserve de respecter les conditions annoncées par l'institution. En outre, le palais de justice ouvre ses portes durant les Journées européennes du patrimoine. Le personnel assure lui-même les visites, souvent agrémentées de compléments conçus pour cette occasion (expositions, animations ou mises en scène...). Le lieu suscite tous les ans l'engouement des visiteurs. Mais « Les prisons font aussi partie de notre patrimoine ${ }^{38}$ "! La prison paloise a accepté d'ouvrir ses portes au public pour les Journées européennes du patrimoine 2019, une première à l'échelle nationale pour un établissement en activité et une expérience qu'elle souhaiterait reconduire. Les visites ont été organisées avec l'aide de l'Inventaire 
du patrimoine de la Ville d'art et d'histoire de Pau et les étudiants en master Patrimoine et musées de l'université de Pau et des pays de l'Adour. Cette rencontre entre un site exceptionnel et un public curieux, certes friand de patrimoine, mais aussi plus simplement d'information, n'a été rendue possible que par l'implication pleine et entière des agents de l'administration pénitentiaire locale et régionale, formateur et surveillants, élèves et directeurs. Il s'est agi de transmettre la mémoire du site, de la prison du XIX ${ }^{e}$ siècle à l'arrivée de la télévision et du téléphone dans les cellules, de faire la promotion des divers métiers de l'administration pénitentiaire et de ressources muséales de l'École nationale d'administration pénitentiaire exposées pour l'occasion. Le tout s'est fait sans voyeurisme, en répondant aux questions portant sur la vie en prison, le respect des individus dans ce cadre, ainsi que les évolutions attendues dans la vie carcérale. Le patrimoine est un prétexte à l'ouverture de lieux d'exception - et les prisons en sont un - qui donnent à comprendre la société au travers de l'histoire de l'architecture, de la ville et du territoire, et n'ont plus vocation à rester coupés du monde extérieur, pour ne pas générer de cliché, mystère ou secret ${ }^{39}$.

Enfin, l'accroissement de l'activité est cause d'une insuffisance des locaux pour l'un comme pour l'autre. Il est prévu de délocaliser une partie des activités du palais (pôle civil du tribunal de grande instance, tribunal de commerce et prud'hommes) dans un ancien orphelinat (une miséricorde), également situé en centre-ville, reconverti en cité judiciaire dont l'ouverture serait prévue en 2022. Ici encore, la présence d'attributs décoratifs témoignant d'une institution d'assistance sociale née sous des auspices religieux dérange et il a été procédé au démontage et à la mise à l'abri d'une croix et d'une statue de la Vierge à l'Enfant. Le transfert de la prison dans un établissement à construire à la périphérie de la ville, dans le quartier dit du Hameau, est acté mais son calendrier est encore incertain. Dans un quartier en pleine reconversion aux portes du cœur de ville, qu'adviendra-t-il des murs de la prison, morceau d'architecture certes authentique et emblématique de la société et de la ville du XIX ${ }^{e}$ siècle, mais bien moins séduisant qu'une « villa anglaise »?

\section{NOTES}

1. Je remercie Christophe Rambert et Caroline Soppelsa pour leurs conseils, Frédéric Massy ainsi que les agents et équipes de l'administration judiciaire et pénitentiaire pour leur soutien précieux.

2. Beau ciel.

3. TAYLOR Alexander, On the curative influence of the climate of Pau and the mineral waters of the Pyrenees, on disease, Londres, John W.Parker, 1842, disponible en anglais en ligne https:// wellcomecollection.org/works/tju348f9/items?canvas=1 [lien valide en octobre 2021].

4. Jean-Baptiste Castetnau, maire de Pau, délibérations du conseil municipal, 24 mai 1855, AC Pau, 1D1/22.

5. BIDOT-GERMA Dominique, DEVOS Cécile \& JULIAT Christine (dir.), Pau, Bordeaux, Ausonius, coll. « Atlas historique des villes de France », 2017. 
6. DEVOS Cécile \& LAROCHE Claude, Pau. Un siècle d'architecture sacrée. 1801-1905, Lyon, Lieux Dits, coll. « Images du patrimoine », 2014.

7. AC Pau, 1A4; voir aussi BIDOT-GERMA Dominique, DEVOS Cécile \& JULIAT Christine (dir.), Pau, p. 215-224.

8. AC Pau, 4Fi148, plan Poublan, 1840 ; AC Pau, 4Fi151 à 4Fi170, plans Perret, 1850-1852, révisés parcellairement en 1863-1864.

9. Mémorial des Pyrénées, 4 février 1846, disponible en ligne https://www.pireneas.fr/ark:/ 12148/bpt6k52329691/f3.item [lien valide en octobre 2021].

10. Lien vers le dossier d'inventaire IA64002335 [lien valide en octobre 2021].

11. AN, F2(II)-BASSES-PYRÉNÉES-3.

12. Délibérations du conseil municipal, 5 août 1847, 13 septembre 1847, 29 janvier 1850 (AC Pau, 1D1/21).

13. Délibération du conseil municipal, 18 avril 1853 (AC Pau, 1D1/20).

14. Pour en savoir plus, voir https://www.dessinsdepau.fr/html/7/selection/page_noticeok.php?Ident=D\&NoticeId=145\&myPos=1 [lien valide en octobre 2021] ; OJALVO David, La Vie à Pau du Premier au Second Empire, catalogue d'exposition (musée des Beaux-Arts de Pau, 24 avril-31 août 1964), Pau, musée des Beaux-Arts, 1964, $\mathrm{n}^{\circ} 32$.

15. Copie du procès-verbal de la pose de la première pierre, $\mathrm{AN}, \mathrm{F}^{21} 1994$.

16. Lettre du préfet des Basses-Pyrénées au ministre de l'Instruction publique, des Cultes et des Beaux-Arts, AN, $\mathrm{F}^{21} 556$.

17. Standardisation du type depuis le grand prix de l'Académie d'architecture de 1782. Voir SZAMBIEN Werner, "Le Langage des palais de justice », in ASSOCIATION FRANÇAISE POUR L'HISTOIRE DE LA JUSTICE, La Justice en ses temples. Regards sur l'architecture judiciaire en France, Paris / Poitiers, Errance / Brissaud, coll. « Art et patrimoine », 1992, p. 71-77.

18. CHÂTEAU-DUTIER Emmanuel, Le Conseil des bâtiments civils et l'administration de l'architecture publique en France, dans la première moitié du XIX ${ }^{e}$ siècle, thèse de doctorat en histoire de l'art, Paris, EPHE, 2016, p. 645-653.

19. CHÂTEAU-DUTIER Emmanuel, Le Conseil des bâtiments civils et l'administration de l'architecture publique en France, dans la première moitié du XIX ${ }^{e}$ siècle, p.656, disponible en ligne https:// tel.archives-ouvertes.fr/tel-02375322 [lien valide en janvier 2022].

20. Voir https://gallica.bnf.fr/ark:/12148/bpt6k86614d [lien valide en octobre 2021].

21. Lien vers le dossier d'inventaire IA64002333.

22. Délibération du conseil municipal, 7 août 1845 (AC Pau, 1D1/19).

23. Délibération du conseil municipal, 7 août 1845 (AC Pau, 1D1/19).

24. Délibération du conseil municipal, 7 août 1845 (AC Pau, 1D1/19).

25. Délibération du conseil municipal, 7 août 1845 (AC Pau, 1D1/19). À Mont-de-Marsan (Landes) par exemple, les trois équipements sont ainsi rassemblés au début du XIX siècle dans l'ancien îlot des Ursulines : BERDOY Anne (dir.), Mont-de-Marsan, Bordeaux, Ausonius, coll. « Atlas historique des villes de France »,2018, p. 232-235 et p. 254. Pierre-Théophile Ségretain associe palais de justice et prison dans la réalisation de ces deux équipements départementaux à Niort (DeuxSèvres) dans le $2^{\mathrm{e}}$ quart du XIX ${ }^{\mathrm{e}}$ siècle : CALLAIS Chantal, « La première prison panoptique demicirculaire en France: une aventure humaine et technique. Pierre-Théophile Ségretain (1798-1864) et la prison de Niort (1828-1853)», in DIEU François \& MBANZOULOU Paul, L'Architecture carcérale, des mots et des murs, actes du colloque éponyme (École nationale d'administration pénitentiaire, Agen, décembre 2010), Toulouse, éditions Privat, 2011, p. 75-92, disponible en ligne https://halshs.archives-ouvertes.fr/halshs-00940421/document [lien valide en octobre 2021]. À Agen (Lot-et-Garonne), palais de justice et prison, contemporains des projets palois et édifiés tous deux dans les années 1860, sont également groupés, simplement séparés par une rue. RENNEVILLE Marc, «Les prisons d'Agen dans la première moitié du XIXe siècle ", Le Lien. Bulletin d'histoire judiciaire et pénitentiaire en Lot-et-Garonne, Agen, archives départementales de Lot- 
et-Garonne / École nationale d'administration pénitentiaire, 2005, disponible en ligne https:// halshs.archives-ouvertes.fr/halshs-01393010/document [lien valide en octobre 2021].

26. Délibération du conseil municipal, 4 août 1856 (AC Pau, 1D122).

27. SOPPELSA Caroline, «Le XIX ${ }^{\mathrm{e}}$ siècle et la question pénitentiaire : un siècle d'expérimentations architecturales dans les prisons de Paris », thèse de doctorat en histoire de l'art (École doctorale Sciences de l'homme et de la société, 12 février 2016), Tours, université de Tours, 2016, p. 326-327.

28. Pour comparaison, la maison d'arrêt de Tarbes (Hautes-Pyrénées) est construite à 650 mètres du tribunal.

29. GRILLON Edme Jean Louis \& NORMAND Alfred, Projets spécimens pour servir à la construction des prisons départementales, Paris, ministère de l'Intérieur / imprimerie Regnier et Dourdet, 1854.

30. FOUCAULT Michel, Surveiller et punir. Naissance de la prison [1975], Paris, Gallimard, coll. «Bibliothèque des Histoires ", 2017, p. 16.

31. DEVOS Cécile, "Recenser, étudier et faire connaître "Saint-Luc" à Pau, un exemple de valorisation du patrimoine asilaire", In situ. Revue des patrimoines, Paris, ministère de la Culture, $n^{\circ}$ 31, 2017 [en ligne] https://journals.openedition.org/insitu/14197 [lien valide en octobre 2021].

32. Registre des ordres du jour du Conseil des Bâtiments civils (4 juillet 1860-7 novembre 1865), AN, $\mathrm{F}^{21} 6381$; F2(II)-BASSES-PYRÉNÉES-3.

33. AN, $\mathrm{F}^{21} 6381$.

34. Voir https://agorha.inha.fr/inhaprod/ark:/54721/00276873 [lien valide en octobre 2021].

35. FOUCAUD Odile, «La prison Saint-Michel. L'Artiste et l'Ingénieur », Atlante, maidécembre 2003, p.114-123, extrait disponible en ligne http://data.over-blog-kiwi.com/ 0/95/22/80/20140317/ob_42ac0e_2003-rapport-odile-foucault.pdf [lien valide en octobre 2021]. Voir aussi FOUCAUD Odile, "L'Asile de Braqueville à Toulouse. Un patrimoine inestimable ", Société archéologique $d u$ Midi de la France, janvier 2004, [en ligne] https:// societearcheologiquedumidi.fr/spip.php?article49 [lien valide en octobre 2021].

36. BLOUET Guillaume-Abel, Projet de prison cellulaire pour 585 condamnés, précédé d'observations sur le système pénitentiaire, Paris, Firmin Didot frères imprimerie, 1843, p. 282-283, disponible en ligne https://gallica.bnf.fr/ark:/12148/bpt6k827799 [lien valide en octobre 2021]. Des idées similaires contemporaines sous-tendent la mise en place de nouveaux asiles d'aliénés, qui dans l'idéal ne sont plus tant des lieux de rétention que des lieux de rédemption par le travail et la prière.

37. SOPPELSA Caroline, « Le XIX ${ }^{\mathrm{e}}$ siècle et la question pénitentiaire : un siècle d'expérimentations architecturales dans les prisons de Paris».

38. Voir https://www.liberation.fr/societe/2014/09/18/les-prisons-font-aussi-partie-de-notrepatrimoine_1103194/ [lien valide en octobre 2021].

39. SOPPELSA Caroline, "Histoire de l'architecture et prisons", Monumental. Le Patrimoine de l'enfermement, 2018-1, p. 8-13.

\section{RÉSUMÉS}

Ancienne capitale de royaume devenue chef-lieu de département, Pau connaît un des tournants de son histoire dans la deuxième moitié du XIX ${ }^{\mathrm{e}}$ siècle, à la faveur de sa renommée nouvelle de 
station touristique et climatique. Son expansion géographique et démographique est encadrée et accompagnée comme il se doit par les différentes autorités exercées sur son territoire. C'est notamment sous le Second Empire que la ville se dote de différents bâtiments propres à héberger et incarner les différentes administrations implantées en ville. Le département des BassesPyrénées se saisit alors des ressources à sa disposition pour programmer une vague de constructions d'édifices publics (nouveaux palais de justice, prisons et asile d'aliénés, trois édifices construits entre 1850 et 1868). La ville constitue à l'époque un foyer propice à la réflexion sur l'architecture de l'administration et les architectes départementaux jouent un rôle non négligeable dans les transformations urbaines mises en œuvre. Il convient ainsi d'interroger la place de l'architecture judiciaire dans le maillage serré des édifices publics destinés à accompagner la croissance du territoire de Pau et des Basses-Pyrénées, qui sembleraient suivre les tendances nationales en les adaptant à leurs modestes moyens. Ces exemples ont conservé leurs fonctions d'origine jusqu'à nos jours ; la mise en valeur de ce patrimoine n'en est que plus éclairante et utile pour une meilleure compréhension de l'histoire urbaine de la cité paloise.

Ancient capital of a kingdom, which has become the chief town of a department, Pau experienced one of the turning points in its history in the second half of the $19^{\text {th }}$ century, thanks to its new reputation as a tourist and climatic resort. Its geographic and demographic expansion is supervised and relayed as it should be by the various authorities exercised on its territory. It was in particular during the Second Empire that the city was to be embellished with various buildings capable of hosting and embodying the various administrations established in the city.

The Basses-Pyrénées department seized the resources at its disposal to program a wave of construction of public buildings (new courthouses, prisons and asylum, three buildings built between 1850 and 1868). At the time, the city was an ideal centre for reflection on administration buildings architecture and the departmental architects played a significant role in the urban transformations implemented. It is therefore necessary to question the place of judicial architecture in the tight network of public buildings intended to support the growth of the territory of Pau and the Basses-Pyrénées, which would seem inclined to follow national trends by adapting to their modest means. These examples have kept their original functions until today; the enhancement of this heritage is all the more interesting and useful for a better understanding of Pau's urban history.

\section{INDEX}

Keywords : courthouse, Pau, prison, departmental architect, asylum, Basses-Pyrénées (department), Latapie (Vincent), Lévy (Gustave), Civil Buildings Council, 19th century architecture

Mots-clés : palais de justice, Pau, prison, architecte départemental, asile d'aliénés, BassesPyrénées, Latapie (Vincent), Lévy (Gustave), Conseil des bâtiments civils, architecture du xixe siècle

\section{AUTEUR}

\section{CÉCILE DEVOS}

Inventaire général du patrimoine culturel

Mission Ville d'art et d'histoire, Ville de Pau

c.devos@ville-pau.fr 